\title{
THE INVESTIGATION OF BIOACTIVE SECONDARY METABOLITES OF THE METHANOL EXTRACT OF ERYNGIUM AMETHYSTINUM
}

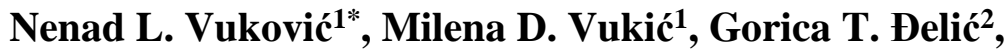 \\ Miroslava M. Kacaniova ${ }^{3}$, Mirjana Cvijovićc \\ ${ }^{1}$ University of Kragujevac, Faculty of Science, Department of Chemistry, P.O. Box 60, \\ 34000 Kragujevac, Serbia \\ ${ }^{2}$ University of Kragujevac, Faculty of Science, Department of Biology and Ecology, \\ P.O. Box 60, 34000 Kragujevac, Serbia \\ ${ }^{3}$ Slovak University of Agriculture in Nitra, Department of Microbiology, \\ Faculty of Biotechnology and Food Science, Nitra, Slovakia \\ ${ }^{4}$ University of Belgrade, Faculty of Medicine, Department for Clinical Microbiology and \\ Immunology, Dr Subotića 1, 11000 Belgrade, Serbia \\ *Corresponding author; E-mail: nvukovic@kg.ac.rs
}

(Received June 22, 2017; Accepted September 10, 2017)

\begin{abstract}
Eryngium amethystinum L. belonging to the Apiaceae family, is a perennial plant distributed in Southeast Europe. Even though this plant is used in traditional medicine, its phytochemical characterization is still incomplete. In this study composition of bioactive constituents of the methanol extract are reported for the first time. By means of the UPLC-LTQ-Orbitrap-MS ${ }^{\mathrm{n}}$ method, altogether sixty-three constituents were characterized: eight hydroxybenzoic acid derivatives (7-13, 32), fifteen cinnamic acid derivatives $(14,17-19,21,24-26,28,30,39-42$ and 44), four flavonoid aglycones $(45,51,52,54)$, twenty-four flavonoid derivatives $(23,27,29,31,33-38,43,46-50,53,55-59,61$ and 62), three coumarin derivatives $(15,16$ and 22$)$ and nine other compounds $(1-6,20,60$ and $63)$.
\end{abstract}

Keywords: metabolic profiling, LC-HRMS, phenolic compounds.

\section{INTRODUCTION}

The genus Eryngium L. is distributed all around the world, and with more than 200 species represents the taxonomically most complex genus of the family Apiaceae (WoRZ, 2004; CALVINO et al., 2007).

Many plants from this genus have valuable ethnopharmacological and nutritional values (FACCIOLA, 1990; ZHANG et al., 2008).

Eryngium amethystinum L., commonly known as amethyst sea holly, is distributed in western and central Serbia on calcareous and arid soil, up to $1600 \mathrm{~m}$ above the sea level. In folk medicine of Southeast Europe E. amethystinym is used for its diuretic and laxative 
properties, in treatment of edemas, urinary ailments, and acidosis. It is also useful as an aid to digestion (FLAMINI et al., 2008).

Previous chemical investigations of Eryngium species revealed the presence of various class of natural products: polyphenols, coumarins, saponins, acetylenes, essential oils (CROWDEN et al., 1969; DRAKE and LAM, 1972; KARTAL et al., 2006; FLAMINI et al., 2008; ZHANG et al., 2008).

Linear ion trap quadrupole-Orbitrap-mass spectrometry (LTQ-Orbitrap-MS) delivers single-stage mass analysis providing molecular mass information, two-stage mass analysis $\left(\mathrm{MS}^{2}\right)$ and multi-stage mass analysis $\left(\mathrm{MS}^{\mathrm{n}}\right)$ delivering structural information. Exact mass measurements and elemental composition assignment are essential for the characterization of small molecules. Accurate mass measurement of the product ions formed in $\mathrm{MS}^{\mathrm{n}}$ experiments facilitates the elucidation of the structures of unknown compounds (TCHOUMTCHOUA et al., 2013)

The aim of the present study was to characterize the secondary bioactive metabolites in the methanol extract of E. amethystinum by using UPLC-HRMS ${ }^{\mathrm{n}}$. The chemical structures of the identified compounds and their glycoside derivatives are important to reveal information on possible bioactive effect of examined plant.

In this paper we presented for the first time chemical profiling and levels of antioxidant activitiy of the methanol extracts of wild growing E. amethystinum.

\section{MATERIALS AND METHODS}

\section{Chemicals and reagents}

LC-MS grade formic acid and acetonitrile were purchased from Thermo Scientific Pierce (Thermo Fisher Scientific, Pierce Biotechnology, Rockford, IL, USA). Water was treated in a Milli-Q water purification system (TGI Pure Water Systems, Brea, CA, USA).

\section{Plant material and sample preparation}

Eryngium amethystinum aerial parts were collected (Jun 2014) in the region of Raška (south Serbia, altitude $350 \mathrm{~m}, 43^{\circ} 30^{\prime} 53^{\prime \prime} \mathrm{N}, 20^{\circ} 37^{\prime} 07^{\prime}$ 'E) and identified at the Department of Biology and Ecology, Faculty of Science in Kragujevac, University of Kragujevac (Serbia). Voucher specimen (17088, BEOU) was deposited at the Department of Botany, Faculty of Biology, University of Belgrade, Serbia.

The collected plant material was air-dried in darkness at ambient temperature. A portion of the sample ( $20 \mathrm{~g}$ ) was ground to a thin powder, and extracted three times (at room temperature, 24h) with HPLC grade methanol $(200 \mathrm{~mL})$. Methanol extracts were combined and evaporated under reduced pressure by means of rotary evaporator at $40{ }^{\circ} \mathrm{C}$. A portion of crude extract $(50 \mathrm{mg})$ was dissolved in methanol, filtered through a membrane filter and used directly for LC-MS analysis.

\section{UPLC-LTQ-Orbitrap-MS for metabolomic analysis}

Chromatographic separations were performed using an ultrahigh-performance liquid chromatography (UPLC) system consisting of a quaternary Accela 600 pump and Accela Autosampler (ThermoFisher Scientific, Bremen, Germany). Analytical column used for separations was a Syncronis $\mathrm{C}_{18}$ column $(100 \times 2.1 \mathrm{~mm}, 1.7 \mu \mathrm{m}$ particle size, ThermoFisher Scientific). The mobile phase consisted of (A) ultrapure water with $1 \%$ formic acid and (B) 
acetonitrile (MS grade). The injection volume for the sample was $10 \mu \mathrm{L}$, elution gradient programme was $5-95 \% \mathrm{~B}$ for $20 \mathrm{~min}$, with the flow rate of $0.3 \mathrm{~mL} / \mathrm{min}$.

The UPLC system was coupled to a linear-trap quadrupole (LTQ) orbitrap hybrid mass spectrometer equipped with a heated-electrospray ionisation probe (HESI-II, ThermoFisher Scientific, Bremen, Germany). The mass spectra were obtained in negative and positive ion modes. Operation parameters were as follows: source voltage $4.5 \mathrm{kV}(4.2 \mathrm{kV}$ in positive mode), capillary voltage $-10 \mathrm{~V}$ ( $42 \mathrm{~V}$ in positive mode), tube lens voltage $-35 \mathrm{~V}$ (110 $\mathrm{V}$ in positive mode), capillary temperature $300{ }^{\circ} \mathrm{C}$, sheath and auxiliary gas flow $\left(\mathrm{N}_{2}\right) 32$ and 8 (arbitrary units), respectively. The MS spectra were acquired by full range acquisition covering 100-1000 m/z, and for the fragmentation study, a data dependent scan was performed by deploying collision-induced dissociation (CID): the normalized collision energy of the CID cell was set to $35 \mathrm{eV}$.

Compounds were identified according to the corresponding spectral characteristics: mass spectra, accurate mass, characteristic fragmentation and characteristic retention time. ThermoFisher Scientific Xcalibur software (Version 2.1) was used for instrument control, data acquisition and data analysis.

\section{RESULTS AND DISCUSSION}

\section{UPLC-LTQ-Orbitrap-MS ${ }^{n}$ identification of secondary metabolites of E. amethystinum}

In the present work, a total of sixty-three compounds were tentatively identified in the sample, mainly phenolic compounds such as: simple phenols, flavonoids, coumarins and other compounds. UPLC-LTQ-Orbitrap $\mathrm{MS}^{\mathrm{n}}$ chromatograms were given in Figure 1 and Figure 2. The tentatively identified metabolites are summarized in Table 1, in negative and positive ionization modes, including retention times, experimental and calculated $[\mathrm{M}-\mathrm{H}]^{-}$and $[\mathrm{M}+\mathrm{H}]^{+}$, errors in $\mathrm{ppm}, \mathrm{MS}^{2}$ and $\mathrm{MS}^{3}$ fragment ions (in negative and positive modes), molecular formula, together with their proposed identities. The compounds were identified by interpreting their mass spectra obtained via LTQ-Orbitrap high resolution mass spectrometer and taking into account the data reported in the literature. 


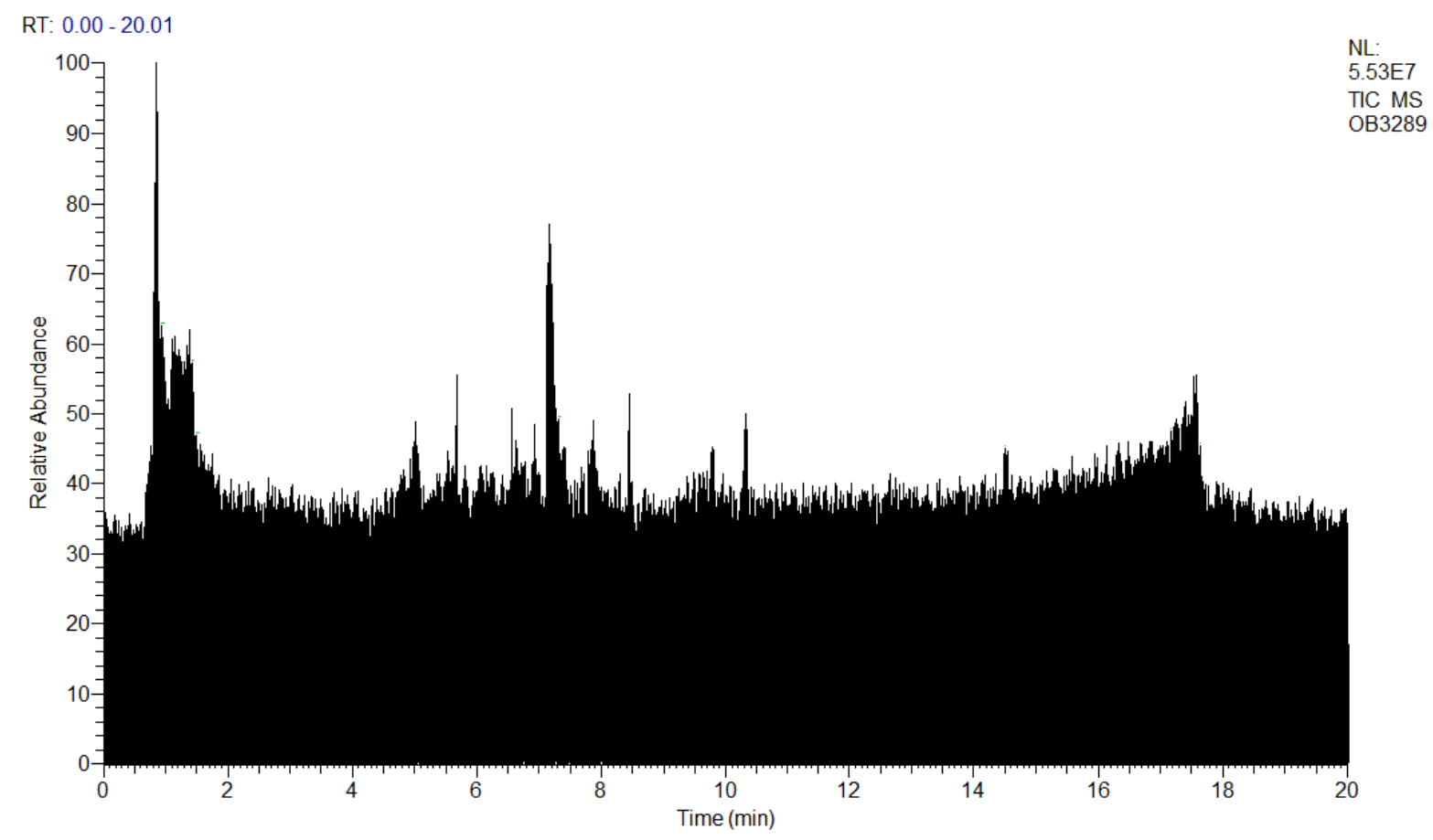

Figure 1. UPLC-LTQ-Orbitrap MS ${ }^{\mathrm{n}}$ in negative mode of methanol extract of E. amethystinum.

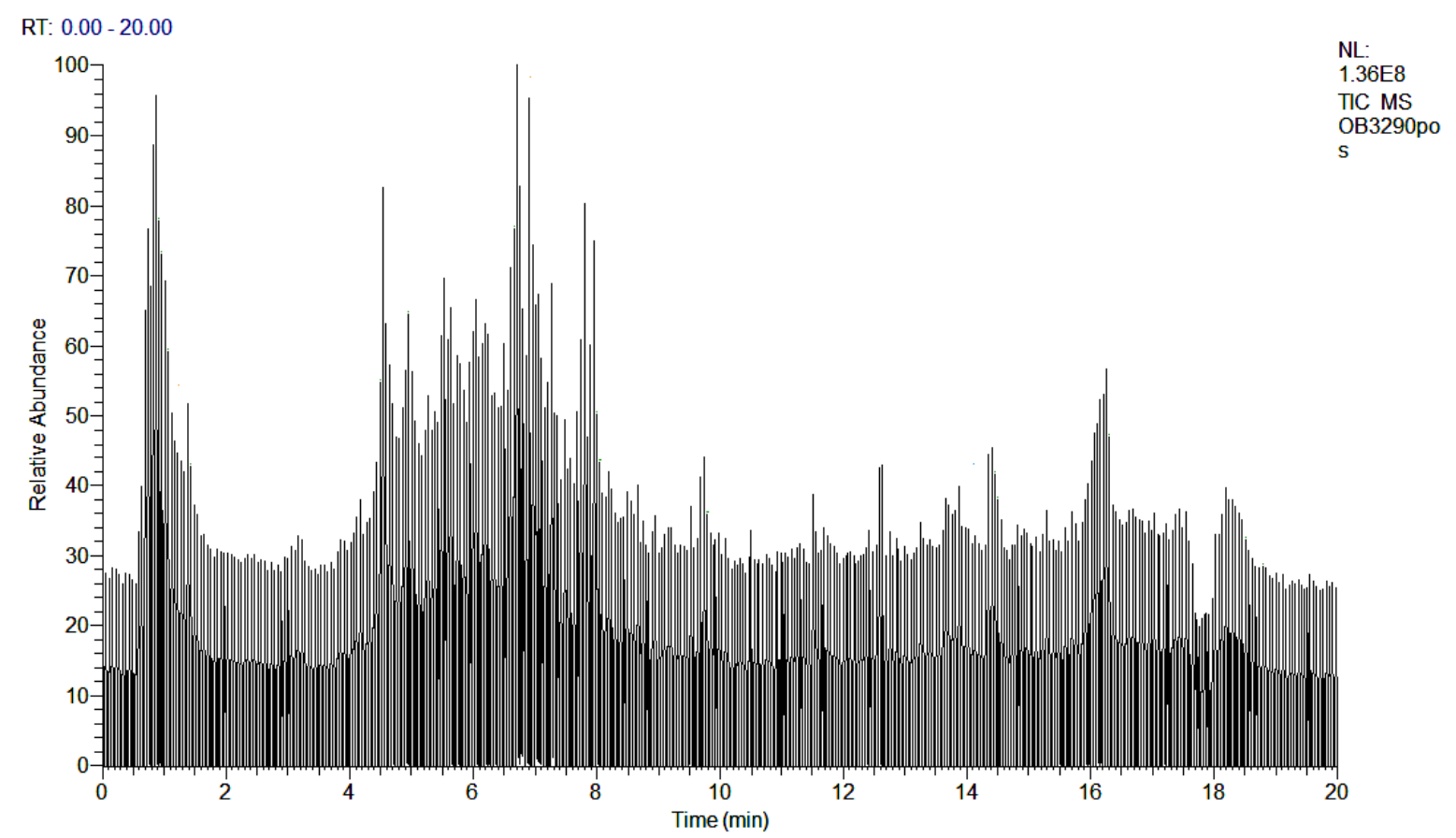

Figure 2. UPLC-LTQ-Orbitrap MS ${ }^{\mathrm{n}}$ in positive mode of methanol extract of E. amethystinum. 
Table 1. List of identified compounds with peak numbers, retention times $\left(\mathrm{t}_{r}\right)$, experimental and calculated masses, error (ppm), $\mathrm{MS}^{2}$ and $\mathrm{MS}^{3}$ fragments, and molecular formulas.

\begin{tabular}{|c|c|c|c|c|c|c|c|}
\hline Peak & Proposed compounds & $\begin{array}{c}\mathbf{t}_{r} \\
(\mathbf{m i n})\end{array}$ & $\begin{array}{l}\text { Exp. } m / z \\
{[\mathrm{M}-\mathrm{H}]^{-1}} \\
{[\mathrm{M}+\mathrm{H}]^{+}}\end{array}$ & $\begin{array}{l}\text { Calc. } m / z \\
{[\mathrm{M}-\mathrm{H}]^{-/}} \\
{[\mathrm{M}+\mathrm{H}]^{+}}\end{array}$ & $\begin{array}{l}\text { Error } \\
(\mathrm{ppm})\end{array}$ & $\begin{array}{c}\text { MS }^{2} \text { fragments }(\%) ; \mathrm{MS}^{3} \text { fragments }(\%) \\
\text { negative mode/ } \\
\text { positive mode }\end{array}$ & $\begin{array}{l}\text { Mol. } \\
\text { formula }\end{array}$ \\
\hline 1 & Gluconic acid & 1.09 & $\begin{array}{l}195.0496 / \\
\text { n. o. }\end{array}$ & $\begin{array}{l}195.0505 / \\
197.0661\end{array}$ & $\begin{array}{l}-1.482 / \\
\text { n. o. }\end{array}$ & $\begin{array}{l}177(31), 159(72), 129(100) ; 85(92), \\
57(100) / \\
\text { n. o. }\end{array}$ & $\mathrm{C}_{6} \mathrm{H}_{12} \mathrm{O}_{7}$ \\
\hline 2 & Quinic acid & 1.15 & $\begin{array}{l}191.0554 / \\
\text { n. } 0 .\end{array}$ & $\begin{array}{l}191.0556 / \\
193.0712\end{array}$ & $\begin{array}{l}1.965 / \\
\text { n. } 0 .\end{array}$ & $\begin{array}{l}127(100) / \\
\text { n. o. }\end{array}$ & $\mathrm{C}_{7} \mathrm{H}_{12} \mathrm{O}_{6}$ \\
\hline 3 & Malic acid & 1.43 & $\begin{array}{l}133.0133 / \\
\text { n. o. }\end{array}$ & $\begin{array}{l}133.0137 / \\
135.0293\end{array}$ & $\begin{array}{l}0.979 / \\
\text { n. o. }\end{array}$ & $\begin{array}{l}115(100), 89(11), 87(10), 71(6) ; 71 \\
(100) / \\
\text { n. o. }\end{array}$ & $\mathrm{C}_{4} \mathrm{H}_{6} \mathrm{O}_{5}$ \\
\hline 4 & Succinic acid & 1.49 & $\begin{array}{l}117.0185 / \\
\text { n. o. }\end{array}$ & $\begin{array}{l}117.0188 / \\
119.0344\end{array}$ & $\begin{array}{l}2.263 / \\
\text { n. o. }\end{array}$ & $\begin{array}{l}59(100) / \\
\text { n. o. }\end{array}$ & $\mathrm{C}_{4} \mathrm{H}_{6} \mathrm{O}_{4}$ \\
\hline 5 & Hydroquinone- $O$-glucoside & 1.56 & $\begin{array}{l}271.0818 / \\
\text { n. } 0 .\end{array}$ & $\begin{array}{l}271.0818 / \\
273.0974\end{array}$ & $\begin{array}{l}1.958 / \\
\text { n. o. }\end{array}$ & $\begin{array}{l}109(100) / \\
\text { n. o. }\end{array}$ & $\mathrm{C}_{12} \mathrm{H}_{16} \mathrm{O}_{7}$ \\
\hline 6 & Hydroxymethylglutaric acid & 2.44 & $\begin{array}{l}161.0446 / \\
163.0594\end{array}$ & $\begin{array}{l}161.0450 / \\
163.0606\end{array}$ & $\begin{array}{l}1.243 / \\
-4.476\end{array}$ & $117(100) /$ & $\mathrm{C}_{6} \mathrm{H}_{10} \mathrm{O}_{5}$ \\
\hline 7 & Glucosyringic acid & 3.17 & $\begin{array}{l}359.0975 / \\
\text { n. } 0 .\end{array}$ & $\begin{array}{l}359.0978 / \\
361.1135\end{array}$ & $\begin{array}{l}0.632 / \\
\text { n. o. }\end{array}$ & $\begin{array}{l}197(100), 179(23) / \\
\text { n. o. }\end{array}$ & $\mathrm{C}_{15} \mathrm{H}_{20} \mathrm{O}_{10}$ \\
\hline 8 & 1-Galloyl-O-glucoside & 3.25 & $\begin{array}{l}331.0644 / \\
\text { n. } 0 .\end{array}$ & $\begin{array}{l}331.0665 / \\
333.0822\end{array}$ & $\begin{array}{l}-4.872 / \\
\text { n. o. }\end{array}$ & $\begin{array}{l}152(100), 124(7) / \\
\text { n. o. }\end{array}$ & $\mathrm{C}_{13} \mathrm{H}_{16} \mathrm{O}_{10}$ \\
\hline 9 & Protocatechuic acid glucoside (isomer) & 3.35 & $\begin{array}{l}315.0721 / \\
\text { n. o. }\end{array}$ & $\begin{array}{l}315.0716 / \\
317.0873\end{array}$ & $\begin{array}{l}3.147 / \\
\text { n. o. }\end{array}$ & $\begin{array}{l}153(100) / \\
\text { n. o. }\end{array}$ & $\mathrm{C}_{13} \mathrm{H}_{16} \mathrm{O}_{9}$ \\
\hline 10 & Syringic acid & 3.49 & $\begin{array}{l}197.0447 / \\
\text { n. o. }\end{array}$ & $\begin{array}{l}197.0450 / \\
199.0606\end{array}$ & $\begin{array}{l}1.320 / \\
\text { n. o. }\end{array}$ & $\begin{array}{l}153(100) / \\
\text { n. o. }\end{array}$ & $\mathrm{C}_{9} \mathrm{H}_{10} \mathrm{O}_{5}$ \\
\hline 11 & Protocatechuic acid & 3.68 & $\begin{array}{l}153.0185 / \\
\text { n. o. }\end{array}$ & $\begin{array}{l}153.0188 / \\
155.0344\end{array}$ & $\begin{array}{l}1.862 / \\
\text { n. o. }\end{array}$ & $\begin{array}{l}109(100) / \\
\text { n. o. }\end{array}$ & $\mathrm{C}_{7} \mathrm{H}_{6} \mathrm{O}_{4}$ \\
\hline 12 & 4-( $\beta$-D-Glucosyloxy)benzoic acid & 3.71 & $\begin{array}{l}299.0755 / \\
\text { n. o. }\end{array}$ & $\begin{array}{l}299.0767 / \\
301.0923\end{array}$ & $\begin{array}{l}-2.153 / \\
\text { n. o. }\end{array}$ & $\begin{array}{l}137(100), 93(27) / \\
\text { n. o. }\end{array}$ & $\mathrm{C}_{13} \mathrm{H}_{16} \mathrm{O}_{8}$ \\
\hline 13 & 1-O-(4-Hydroxy-3,5-dimethoxybenzoyl)-glucoside & 4.17 & $359.0966 /$ & $359.0978 /$ & $-1.819 /$ & 197 (100), 182 (3); 182 (100), 153 & $\mathrm{C}_{15} \mathrm{H}_{20} \mathrm{O}_{10}$ \\
\hline
\end{tabular}




\begin{tabular}{|c|c|c|c|c|c|c|c|}
\hline & & & 361.1122 & 361.1135 & -1.892 & $\begin{array}{l}(23), 138(8) / \\
\text { n. o. }\end{array}$ & \\
\hline 14 & Chlorogenic acid (isomer) & 4.69 & $\begin{array}{l}353.0856 / \\
355.1017\end{array}$ & $\begin{array}{l}353.0873 / \\
355.1029\end{array}$ & $\begin{array}{l}-3.055 / \\
-1.911\end{array}$ & $\begin{array}{l}191(100), 179(39), 135(7) ; 173(81), \\
127(92), 111(31), 93(47), 85(100) / \\
309(11), 165(100), 147(77) ; 147 \\
(100)\end{array}$ & $\mathrm{C}_{16} \mathrm{H}_{18} \mathrm{O}_{9}$ \\
\hline 15 & Esculetin-6-O-glucoside & 4.72 & $\begin{array}{l}339.0710 / \\
341.0864\end{array}$ & $\begin{array}{l}339.0716 / \\
341.0873\end{array}$ & $\begin{array}{l}-0.231 / \\
-0.817\end{array}$ & $\begin{array}{l}177(100) / \\
\text { n. o. }\end{array}$ & $\mathrm{C}_{15} \mathrm{H}_{16} \mathrm{O}_{9}$ \\
\hline 16 & Scopoletin-7-O-glucuronide & 4.76 & $\begin{array}{l}367.0659 / \\
369.0805\end{array}$ & $\begin{array}{l}367.0665 / \\
369.0822\end{array}$ & $\begin{array}{l}-0.145 / \\
-3.124\end{array}$ & $\begin{array}{l}191(100) / \\
\text { n. o. }\end{array}$ & $\mathrm{C}_{16} \mathrm{H}_{16} \mathrm{O}_{10}$ \\
\hline 17 & Chlorogenic acid (isomer) & 4.86 & $\begin{array}{l}353.0855 / \\
355.1016\end{array}$ & $\begin{array}{l}353.0873 / \\
355.1029\end{array}$ & $\begin{array}{l}-3.394 / \\
-2.080\end{array}$ & $\begin{array}{l}191(100), 179(<3) ; 173(83), 127 \\
(96), 111(30), 93(49), 85(100) / \\
309(10), 165(100), 147(63) ; 147 \\
(100)\end{array}$ & $\mathrm{C}_{16} \mathrm{H}_{18} \mathrm{O}_{9}$ \\
\hline 18 & 4-O-Glucosyl- $p$-coumaric acid & 4.97 & $\begin{array}{l}325.0910 / \\
327.1078\end{array}$ & $\begin{array}{l}325.0923 / \\
327.1080\end{array}$ & $\begin{array}{l}-2.350 / \\
1.150\end{array}$ & $\begin{array}{l}163(100) / \\
\text { n. o. }\end{array}$ & $\mathrm{C}_{15} \mathrm{H}_{18} \mathrm{O}_{8}$ \\
\hline 19 & Chlorogenic acid (isomer) & 5.06 & $\begin{array}{l}353.0851 / \\
355.1019\end{array}$ & $\begin{array}{l}353.0873 / \\
355.1029\end{array}$ & $\begin{array}{l}-4.612 / \\
-1.376\end{array}$ & $\begin{array}{l}191(100), 179(15), 161(3) ; 173(84), \\
127(94), 111(33), 93(52), 85(100) / \\
309(11), 165(100), 147(87) ; 147 \\
(100)\end{array}$ & $\mathrm{C}_{16} \mathrm{H}_{18} \mathrm{O}_{9}$ \\
\hline 20 & Nonyl- $O$-maltoside & 5.10 & $\begin{array}{l}467.2493 / \\
469.2637\end{array}$ & $\begin{array}{l}467.2492 / \\
469.2649\end{array}$ & $\begin{array}{l}1.373 / \\
-1.360\end{array}$ & $\begin{array}{l}\text { n. o. / } \\
451 \text { (7), } 307 \text { (100), } 289 \text { (27), } 271 \text { (29); } \\
288(94), 271(100)\end{array}$ & $\mathrm{C}_{21} \mathrm{H}_{40} \mathrm{O}_{11}$ \\
\hline 21 & Caffeic acid & 5.52 & $\begin{array}{l}179.0339 / \\
\text { n. o. }\end{array}$ & $\begin{array}{l}179.0344 / \\
181.0501\end{array}$ & $\begin{array}{l}-0.085 / \\
\text { n. o. }\end{array}$ & $\begin{array}{l}135(100) / \\
\text { n. o. }\end{array}$ & $\mathrm{C}_{9} \mathrm{H}_{8} \mathrm{O}_{4}$ \\
\hline 22 & (4-Methylumbelliferone)-7-O-glucoside & 5.69 & $\begin{array}{l}337.0924 / \\
339.1071\end{array}$ & $\begin{array}{l}337.0923 / \\
339.1080\end{array}$ & $\begin{array}{l}1.828 / \\
-0.867\end{array}$ & $\begin{array}{l}175(100) / \\
177(100)\end{array}$ & $\mathrm{C}_{16} \mathrm{H}_{18} \mathrm{O}_{8}$ \\
\hline 23 & Quercetin-3-O-rutinoside & 5.72 & $\begin{array}{l}609.1461 / \\
611.1601\end{array}$ & $\begin{array}{l}609.1456 / \\
611.1612\end{array}$ & $\begin{array}{l}1.722 / \\
-0.918\end{array}$ & $\begin{array}{l}463(6), 301(100), 300(32) / \\
\text { n. o. }\end{array}$ & $\mathrm{C}_{27} \mathrm{H}_{30} \mathrm{O}_{16}$ \\
\hline 24 & 5-O-p-Coumaroylquinic acid & 5.76 & $\begin{array}{l}337.0911 / \\
339.1069\end{array}$ & $\begin{array}{l}337.0923 / \\
339.1080\end{array}$ & $\begin{array}{l}-2.177 / \\
-1.516\end{array}$ & $\begin{array}{l}191(20), 163(100) / \\
\text { n. o. }\end{array}$ & $\mathrm{C}_{16} \mathrm{H}_{18} \mathrm{O}_{8}$ \\
\hline 25 & Feruloylquinic acid (isomer) & 5.94 & $\begin{array}{l}367.1017 / \\
369.1176\end{array}$ & $\begin{array}{l}367.1029 / \\
369.1186\end{array}$ & $\begin{array}{l}-1.848 / \\
-1.080\end{array}$ & $\begin{array}{l}191(100), 173(<2) ; 173(74), 171 \\
(31), 127(100), 111(41), 93(60), 85 \\
(92) /\end{array}$ & $\mathrm{C}_{17} \mathrm{H}_{20} \mathrm{O}_{9}$ \\
\hline
\end{tabular}




\begin{tabular}{|c|c|c|c|c|c|c|c|}
\hline 26 & Feruloylquinic acid (isomer) & 6.14 & $\begin{array}{l}367.1013 / \\
369.1177\end{array}$ & $\begin{array}{l}367.1029 / \\
369.1186\end{array}$ & $\begin{array}{l}-2.993 / \\
-0.890\end{array}$ & $\begin{array}{l}191(100), 173(5) ; 173(72), 127 \\
(100), 111(54), 93(51), 85(87) / \\
\text { n. o. }\end{array}$ & $\mathrm{C}_{17} \mathrm{H}_{20} \mathrm{O}_{9}$ \\
\hline 27 & Quercetin-3-O-neohesperidoside & 6.26 & $\begin{array}{l}609.1452 / \\
611.1599\end{array}$ & $\begin{array}{l}609.1456 / \\
611.1612\end{array}$ & $\begin{array}{l}0.310 / \\
-1.229\end{array}$ & $\begin{array}{l}301(100) ; 273(14), 271(71), 193(7), \\
179(100), 151(62) / \\
465(19), 303(100) ; 285(41), 257 \\
(100), 229(88), 165)\end{array}$ & $\mathrm{C}_{27} \mathrm{H}_{30} \mathrm{O}_{16}$ \\
\hline 28 & 3'-O-Glucopyranosyl rosmarinic acid & 6.34 & $\begin{array}{l}521.1284 / \\
523.1439\end{array}$ & $\begin{array}{l}521.1295 / \\
523.1452\end{array}$ & $\begin{array}{l}-1.165 / \\
-1.333\end{array}$ & $\begin{array}{l}359(100), 197(7), 161(4) ; 223(14), \\
197(92), 179(49), 161(100) / \\
\text { n. o. }\end{array}$ & $\mathrm{C}_{24} \mathrm{H}_{26} \mathrm{O}_{13}$ \\
\hline 29 & Kaempferol-3- $O$-neohesperidoside & 6.36 & $\begin{array}{l}593.1500 / \\
595.1651\end{array}$ & $\begin{array}{l}593.1506 / \\
595.1663\end{array}$ & $\begin{array}{l}-0.129 / \\
-1.086\end{array}$ & $\begin{array}{l}447(17), 429(49), 285(100) ; 255 \\
(100), 227(10) / \\
449(17), 287(100) ; 258(42), 241 \\
(100), 213(72), 165(94), 153(53)\end{array}$ & $\mathrm{C}_{27} \mathrm{H}_{30} \mathrm{O}_{15}$ \\
\hline 30 & $p$-Coumaric acid & 6.38 & $\begin{array}{l}163.0388 / \\
\text { n. o. }\end{array}$ & $\begin{array}{l}163.0395 / \\
165.0552\end{array}$ & $\begin{array}{l}-1.292 / \\
\text { n. o }\end{array}$ & $\begin{array}{l}119(100) ; 92(40), 91(100) / \\
\text { n. o }\end{array}$ & $\mathrm{C}_{9} \mathrm{H}_{8} \mathrm{O}_{3}$ \\
\hline 31 & Quercetin-3-O-glucoside & 6.47 & $\begin{array}{l}463.0859 / \\
465.1021\end{array}$ & $\begin{array}{l}463.0877 / \\
465.1033\end{array}$ & $\begin{array}{l}-2.510 / \\
-1.446\end{array}$ & $\begin{array}{l}301(100) ; 271(31), 255(22), 179 \\
(100), 150(49) / \\
303(100) ; 285(41), 257(100), 247 \\
(31), 229(63), 165(48)\end{array}$ & $\mathrm{C}_{21} \mathrm{H}_{20} \mathrm{O}_{12}$ \\
\hline 32 & Methyl 4-hydroxybenzoate & 6.52 & $\begin{array}{l}151.0390 / \\
153.0540\end{array}$ & $\begin{array}{l}151.0395 / \\
153.0552\end{array}$ & $\begin{array}{l}0.128 / \\
-4.251\end{array}$ & $\begin{array}{l}136(100) ; 108(23), 92(100) / \\
\text { n. o. }\end{array}$ & $\mathrm{C}_{8} \mathrm{H}_{8} \mathrm{O}_{3}$ \\
\hline 33 & Kaempferol- $O$-rhamnoside- $O$-xyloside & 6.55 & $\begin{array}{l}563.1401 / \\
565.1543\end{array}$ & $\begin{array}{l}563.1401 / \\
565.1557\end{array}$ & $\begin{array}{l}0.973 / \\
-1.560\end{array}$ & $\begin{array}{l}\text { n. o. / } \\
433(100), 419 \text { (13), } 287 \text { (11); } 287 \\
(100)\end{array}$ & $\mathrm{C}_{26} \mathrm{H}_{28} \mathrm{O}_{14}$ \\
\hline 34 & Kaempferol-3-O-glucoside & 6.57 & $\begin{array}{l}447.0927 / \\
449.1072\end{array}$ & $\begin{array}{l}447.0927 / \\
449.1084\end{array}$ & $\begin{array}{l}1.235 / \\
-1.465\end{array}$ & $\begin{array}{l}285(100) / \\
287(100)\end{array}$ & $\mathrm{C}_{21} \mathrm{H}_{20} \mathrm{O}_{11}$ \\
\hline 35 & Isorhamnetin- $O$-rutinoside & 6.63 & $\begin{array}{l}623.1614 / \\
625.1751\end{array}$ & $\begin{array}{l}623.1612 / \\
625.1769\end{array}$ & $\begin{array}{l}1.154 / \\
-1.921\end{array}$ & $\begin{array}{l}315(100), 300(12) ; 300(100), 285 \\
(27) / \\
\text { n. o. }\end{array}$ & $\mathrm{C}_{28} \mathrm{H}_{32} \mathrm{O}_{16}$ \\
\hline 36 & Isorhamnetin- $O$-glucoside & 6.75 & $\begin{array}{l}477.1013 / \\
479.1175\end{array}$ & $\begin{array}{l}477.1013 / \\
479.1190\end{array}$ & $\begin{array}{l}-3.128 / \\
-1.905\end{array}$ & $\begin{array}{l}315(100) ; 300(100) / \\
317(100) ; 302(100)\end{array}$ & $\mathrm{C}_{22} \mathrm{H}_{22} \mathrm{O}_{12}$ \\
\hline 37 & Syringetin- $O$-glucoside & 6.86 & $507.1127 /$ & $507.1139 /$ & $-1.276 /$ & n. o. / & $\mathrm{C}_{23} \mathrm{H}_{24} \mathrm{O}_{13}$ \\
\hline
\end{tabular}




\begin{tabular}{|c|c|c|c|c|c|c|c|}
\hline & & & 509.1282 & 509.1295 & -1.566 & 347 (100); $332(100)$ & \\
\hline 38 & Luteolin-7-O-glucoside & 6.93 & $\begin{array}{l}447.0903 / \\
449.1065\end{array}$ & $\begin{array}{l}447.0927 / \\
449.1084\end{array}$ & $\begin{array}{l}-4.289 / \\
-3.023\end{array}$ & $\begin{array}{l}285 \text { (100); } 255 \text { (100), } 227(11) / \\
\text { n. o. }\end{array}$ & $\mathrm{C}_{21} \mathrm{H}_{20} \mathrm{O}_{11}$ \\
\hline 39 & Cichoric acid & 6.97 & $\begin{array}{l}473.0706 / \\
\text { n. o. }\end{array}$ & $\begin{array}{l}473.0720 / \\
475.0877\end{array}$ & $\begin{array}{l}-1.886 / \\
\text { n. o. }\end{array}$ & $\begin{array}{l}311(52), 293(100), 179(10) ; 275 \\
(19), 219(100) / \\
\text { n. o. }\end{array}$ & $\mathrm{C}_{22} \mathrm{H}_{18} \mathrm{O}_{12}$ \\
\hline 40 & Dicaffeoylquinic acid (isomer) & 7.06 & $\begin{array}{l}515.1191 / \\
517.1333\end{array}$ & $\begin{array}{l}515.1190 / \\
517.1346\end{array}$ & $\begin{array}{l}1.315 / \\
-1.455\end{array}$ & $\begin{array}{l}353(100), 235(18), 179(8) / \\
\text { n. o. }\end{array}$ & $\mathrm{C}_{25} \mathrm{H}_{24} \mathrm{O}_{12}$ \\
\hline 41 & Dicaffeoylquinic acid (isomer) & 7.11 & $\begin{array}{l}515.1194 / \\
517.1332\end{array}$ & $\begin{array}{l}515.1190 / \\
517.1346\end{array}$ & $\begin{array}{l}2.034 / \\
-1.571\end{array}$ & $\begin{array}{l}353(100), 335(3) / \\
\text { n. o. }\end{array}$ & $\mathrm{C}_{25} \mathrm{H}_{24} \mathrm{O}_{12}$ \\
\hline 42 & Rosmarinic acid & 7.09 & $\begin{array}{l}359.0750 / \\
361.0915\end{array}$ & $\begin{array}{l}359.0767 / \\
361.0923\end{array}$ & $\begin{array}{l}-3.325 / \\
-0.759\end{array}$ & $\begin{array}{l}197(31), 179(39), 161(100) / \\
\text { n. o. }\end{array}$ & $\mathrm{C}_{18} \mathrm{H}_{16} \mathrm{O}_{8}$ \\
\hline 43 & Isorhamnetin- $O$-pentoside & 7.26 & $\begin{array}{l}447.0907 / \\
449.1068\end{array}$ & $\begin{array}{l}447.0927 / \\
449.1084\end{array}$ & $\begin{array}{l}-3.395 / \\
-2.422\end{array}$ & $\begin{array}{l}315(100), 272(11) ; 300(100) / \\
317(100) ; 302(100)\end{array}$ & $\mathrm{C}_{21} \mathrm{H}_{20} \mathrm{O}_{11}$ \\
\hline 44 & Dicaffeoylquinic acid (isomer) & 7.32 & $\begin{array}{l}515.1195 / \\
517.1335\end{array}$ & $\begin{array}{l}515.1190 / \\
517.1346\end{array}$ & $\begin{array}{l}2.849 / \\
-1.107\end{array}$ & $\begin{array}{l}353(70), 235(75), 179(100) / \\
\text { n. o. }\end{array}$ & $\mathrm{C}_{25} \mathrm{H}_{24} \mathrm{O}_{12}$ \\
\hline 45 & Isorhamnetin & 7.34 & $\begin{array}{l}315.0495 / \\
317.0652\end{array}$ & $\begin{array}{l}315.0505 / \\
317.0661\end{array}$ & $\begin{array}{l}-1.362 / \\
-1.322\end{array}$ & $\begin{array}{l}\text { n. o. / } \\
302(100) ; 301(38), 274(100), 256 \\
(62), 228(53), 152(7), 124(3)\end{array}$ & $\mathrm{C}_{16} \mathrm{H}_{12} \mathrm{O}_{7}$ \\
\hline 46 & Kaempferol-3-O-pentoside & 7.47 & $\begin{array}{l}417.0800 / \\
419.0967\end{array}$ & $\begin{array}{l}417.0822 / \\
419.0978\end{array}$ & $\begin{array}{l}-3.916 / \\
-1.344\end{array}$ & $\begin{array}{l}285(100) / \\
287(100)\end{array}$ & $\mathrm{C}_{20} \mathrm{H}_{18} \mathrm{O}_{10}$ \\
\hline 47 & Kaempferol-3-O-( $p$-coumaroyl)-glucoside (isomer) & 8.19 & $\begin{array}{l}593.1286 / \\
595.1437\end{array}$ & $\begin{array}{l}593.1295 / \\
595.1452\end{array}$ & $\begin{array}{l}-0.619 / \\
-1.575\end{array}$ & $\begin{array}{l}\text { n. o. / } \\
449(14), 309 \text { (67), } 291(27), 287 \text { (100) }\end{array}$ & $\mathrm{C}_{30} \mathrm{H}_{26} \mathrm{O}_{13}$ \\
\hline 48 & Kaempferol-3-O-(feruloyl)-glucoside (isomer) & 8.29 & $\begin{array}{l}623.1370 / \\
625.1541\end{array}$ & $\begin{array}{l}623.1401 / \\
625.1557\end{array}$ & $\begin{array}{l}-4.128 / \\
-1.715\end{array}$ & $\begin{array}{l}\text { n. o. / } \\
339 \text { (70), } 321 \quad(22), 287 \quad(100), 195 \\
(18), 177(38)\end{array}$ & $\mathrm{C}_{31} \mathrm{H}_{28} \mathrm{O}_{14}$ \\
\hline 49 & Kaempferol-3-O-(feruloyl)-glucoside (isomer) & 8.34 & $\begin{array}{l}623.1423 / \\
625.1544\end{array}$ & $\begin{array}{l}623.1401 / \\
625.1557\end{array}$ & $\begin{array}{l}1.169 / \\
-1.219\end{array}$ & $\begin{array}{l}\text { n. o. / } \\
339 \text { (67), } 321 \text { (24), } 287 \text { (100), } 195 \\
(19), 177(45)\end{array}$ & $\mathrm{C}_{31} \mathrm{H}_{28} \mathrm{O}_{14}$ \\
\hline 50 & Kaempferol-3-O-(p-coumaroyl)-glucoside (isomer) & 8.38 & $\begin{array}{l}593.1293 / \\
595.1437\end{array}$ & $\begin{array}{l}593.1295 / \\
595.1452\end{array}$ & $\begin{array}{l}0.511 / \\
-1.474\end{array}$ & $\begin{array}{l}\text { n. o. / } \\
449 \text { (9), } 309 \text { (5), } 291 \text { (3), } 287 \text { (100) }\end{array}$ & $\mathrm{C}_{30} \mathrm{H}_{26} \mathrm{O}_{13}$ \\
\hline 51 & Luteolin & 8.46 & $\begin{array}{l}285.0396 / \\
287.0547\end{array}$ & $\begin{array}{l}285.0399 / \\
287.0556\end{array}$ & $\begin{array}{l}0.756 / \\
-1.325 \\
\end{array}$ & $\begin{array}{l}241(100), 199(78), 175(82) / \\
\text { n. o. }\end{array}$ & $\mathrm{C}_{15} \mathrm{H}_{10} \mathrm{O}_{6}$ \\
\hline
\end{tabular}




\begin{tabular}{|c|c|c|c|c|c|c|c|}
\hline 52 & Quercetin & 8.52 & $\begin{array}{l}301.0336 / \\
303.0493\end{array}$ & $\begin{array}{l}301.0348 / \\
303.0505\end{array}$ & $\begin{array}{l}-2.156 / \\
-2.109\end{array}$ & $\begin{array}{l}179(100), 151(63), 121(12) / \\
\text { n. o. }\end{array}$ & $\mathrm{C}_{15} \mathrm{H}_{10} \mathrm{O}_{7}$ \\
\hline 53 & Quercetin-3-O-(di- $p$-coumaroyl)-glucoside (isomer) & 9.35 & $\begin{array}{l}755.1666 / \\
757.1748\end{array}$ & $\begin{array}{l}755.1612 / \\
757.1769\end{array}$ & $\begin{array}{l}7.904 / \\
-1.996\end{array}$ & $\begin{array}{l}\text { n. o. / } \\
455(100), 437 \quad(12) ; 437 \quad(56), 291 \\
(14), 147(100)\end{array}$ & $\mathrm{C}_{39} \mathrm{H}_{32} \mathrm{O}_{16}$ \\
\hline 54 & Kaempferol & 9.50 & $\begin{array}{l}285.0390 / \\
287.0547\end{array}$ & $\begin{array}{l}285.0399 / \\
287.0556\end{array}$ & $\begin{array}{l}-1.384 / \\
-0.922\end{array}$ & $\begin{array}{l}257(11), 229 \text { (100), } 213 \text { (67), } 151 \\
(62) / \\
\text { n. o. }\end{array}$ & $\mathrm{C}_{15} \mathrm{H}_{10} \mathrm{O}_{6}$ \\
\hline 55 & Quercetin-3-O-(di- $p$-coumaroyl)-glucoside (isomer) & 9.57 & $\begin{array}{l}\text { n. o. / } \\
757.1752\end{array}$ & $\begin{array}{l}755.1612 / \\
757.1769\end{array}$ & $\begin{array}{l}\text { n. } 0 . / \\
-1.507\end{array}$ & $\begin{array}{l}\text { n. o. / } \\
455(100), 437 \quad(15) ; 437 \quad(62), 291 \\
(16), 147(100)\end{array}$ & $\mathrm{C}_{39} \mathrm{H}_{32} \mathrm{O}_{16}$ \\
\hline 56 & $\begin{array}{l}\text { Kaempferol-3-O-(di- } p \text {-coumaroyl)-glucoside } \\
\text { (isomer) }\end{array}$ & 9.73 & $\begin{array}{l}739.1678 / \\
741.1803\end{array}$ & $\begin{array}{l}739.1663 / \\
741.1819\end{array}$ & $\begin{array}{l}2.765 / \\
-1.480\end{array}$ & $\begin{array}{l}\text { n. o. / } \\
455(100), 437 \text { (8), } 287(<3) ; 437 \text { (39), } \\
147 \text { (100) }\end{array}$ & $\mathrm{C}_{39} \mathrm{H}_{32} \mathrm{O}_{15}$ \\
\hline 57 & $\begin{array}{l}\text { Kaempferol-3-O-( } p \text {-coumaroyl)-(feruloyl)-glucoside } \\
\text { (isomer) }\end{array}$ & 9.83 & $\begin{array}{l}769.1747 / \\
771.1909\end{array}$ & $\begin{array}{l}769.1769 / \\
771.1925\end{array}$ & $\begin{array}{l}-2.043 / \\
-1.428\end{array}$ & $\begin{array}{l}\text { n. o. / } \\
485 \quad(100), 467 \quad(9) ; 467 \quad(52), 177 \\
(100), 147(19)\end{array}$ & $\mathrm{C}_{40} \mathrm{H}_{34} \mathrm{O}_{16}$ \\
\hline 58 & $\begin{array}{l}\text { Kaempferol-3-O-(di-p-coumaroyl)-glucoside } \\
\text { (isomer) }\end{array}$ & 9.92 & $\begin{array}{l}739.1695 / \\
741.1799\end{array}$ & $\begin{array}{l}739.1663 / \\
741.1819\end{array}$ & $\begin{array}{l}5.064 / \\
-1.979\end{array}$ & $\begin{array}{l}\text { n. o. / } \\
455 \text { (100), } 437 \text { (9), } 287 \text { (11); } 437 \text { (32), } \\
147 \text { (100) }\end{array}$ & $\mathrm{C}_{39} \mathrm{H}_{32} \mathrm{O}_{15}$ \\
\hline 59 & $\begin{array}{l}\text { Kaempferol-3-O-( } p \text {-coumaroyl)-(feruloyl)-glucoside } \\
\text { (isomer) }\end{array}$ & 10.02 & $\begin{array}{l}769.1746 / \\
771.1906\end{array}$ & $\begin{array}{l}769.1769 / \\
771.1925\end{array}$ & $\begin{array}{l}-2.277 / \\
-1.830\end{array}$ & $\begin{array}{l}\text { n. o. / } \\
485 \quad(100), 467 \quad(9) ; 467 \quad(32), 177 \\
(100), 147(16)\end{array}$ & $\mathrm{C}_{40} \mathrm{H}_{34} \mathrm{O}_{16}$ \\
\hline 60 & Dihydroxyhexadecanoic acid & 10.07 & $\begin{array}{l}287.2208 / \\
289.2368\end{array}$ & $\begin{array}{l}287.2222 / \\
289.2379\end{array}$ & $\begin{array}{l}-2.980 / \\
-1.680\end{array}$ & $\begin{array}{l}\text { n. o. / } \\
271 \text { (100), } 253 \text { (11); } 253 \text { (100), } 235 \\
\text { (54) }\end{array}$ & $\mathrm{C}_{16} \mathrm{H}_{32} \mathrm{O}_{4}$ \\
\hline 61 & $\begin{array}{l}\text { Kaempferol-3-O-(di- } p \text {-coumaroyl)-glucoside } \\
\text { (isomer) }\end{array}$ & 10.12 & $\begin{array}{l}739.1685 / \\
741.1798\end{array}$ & $\begin{array}{l}739.1663 / \\
741.1819\end{array}$ & $\begin{array}{l}3.671 / \\
-1.587\end{array}$ & $\begin{array}{l}\text { n. o. / } \\
455 \text { (100), } 437 \text { (8), } 287 \text { (11); } 437 \text { (32), } \\
147 \text { (100) }\end{array}$ & $\mathrm{C}_{39} \mathrm{H}_{32} \mathrm{O}_{15}$ \\
\hline 62 & Isorhamnetin- $O$-( $p$-coumaroyl)-(feruloyl)-glucoside & 10.18 & $\begin{array}{l}\text { n. o. / } \\
801.2009\end{array}$ & $\begin{array}{l}\text { 799.1874. / } \\
801.2031\end{array}$ & $\begin{array}{l}\text { n. o. / } \\
-2.067\end{array}$ & $\begin{array}{l}\text { n. o. / } \\
485 \text { (100), } 467 \text { (5); } 467 \text { (49), } 177 \text { (100) }\end{array}$ & $\mathrm{C}_{41} \mathrm{H}_{36} \mathrm{O}_{17}$ \\
\hline 63 & Hexadecanedioic acid & 10.49 & $\begin{array}{l}\text { n. o./ } \\
287.2211\end{array}$ & $\begin{array}{l}285.2066 / \\
287.2222\end{array}$ & $\begin{array}{l}\text { n. } 0 . / \\
-2.040\end{array}$ & $\begin{array}{l}\text { n. o./ } \\
269(100) ; 251(100)\end{array}$ & $\mathrm{C}_{16} \mathrm{H}_{30} \mathrm{O}_{4}$ \\
\hline
\end{tabular}




\section{Benzoic acid and hydroxycinnamic derivatives}

As can be seen from Table 1, twenty-three compounds were identified. Among them, eight hydroxybenzoic acid and fifteen cinnamic acid derivatives were found.

Compound 7 gave a $[\mathrm{M}-\mathrm{H}]^{-}$ion at $m / z, 359$ and its $\mathrm{MS}^{2}$ fragmentation resulted in

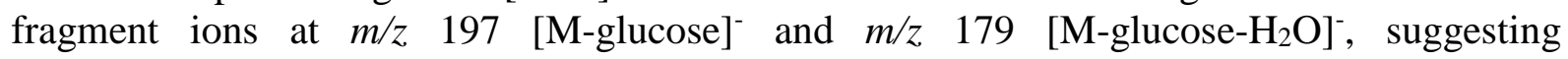
glucosyringic acid. Syringic acid (10) was identified on the basis of $[\mathrm{M}-\mathrm{H}]^{-}$ion at $\mathrm{m} / \mathrm{z} 197$ and characteristic loss of $\mathrm{CO}_{2}$ during formation of $\mathrm{MS}^{2}$ ion at $\mathrm{m} / z$ 153. Compound $\mathbf{8}$ exhibited an [M-H] $]^{-}$ion at $\mathrm{m} / \mathrm{z}, 331$, and its fragmentation showed base peak ion at $\mathrm{m} / z, 152$ [M-glucose$\mathrm{OH}]^{-}$. This compound corresponds to a 1-galloyl-O-glucoside. Protocatechuic acid glucoside, as compound 9, yielded a $[\mathrm{M}-\mathrm{H}]^{-}$ion at $m / z, 315$ and its $\mathrm{MS}^{2}$ fragmentation gave the aglycone fragment ion at $\mathrm{m} / \mathrm{z} 153$ (-162 Da, loss of glucose residue). Elimination of $44 \mathrm{Da}$ from deprotonated molecular ion and $\mathrm{MS}^{2}$ ion at $\mathrm{m} / \mathrm{z}$ 109, indicated that compound $\mathbf{1 1}$ was protocatechuic acid. Compound 12, 4-( $\beta$-D-glucosyloxy)benzoic acid, was characterized by loss of $162 \mathrm{Da}$ from $[\mathrm{M}-\mathrm{H}]^{-}$ion at $m / z 299$, as well as from $\mathrm{MS}^{2}$ ion at $m / z, 93$ [M-glucose$\left.\mathrm{CO}_{2}\right]^{-}$. After examination of $\mathrm{MS}^{2}$ (ion at $\mathrm{m} / \mathrm{z}$ 197, loss of glucose residue) and $\mathrm{MS}^{3}$ spectra (ion at $\mathrm{m} / \mathrm{z} 182$, loss of methyl group; ion at $\mathrm{m} / \mathrm{z} 153$, loss of $\mathrm{CO}_{2}$ ), compound 13 was assigned as 1-O-(4-hydroxy-3,5-dimethoxybenzoyl)-glucoside. The molecular ions in negative and positive modes indicated that compound 32 had molecular formula $\mathrm{C}_{8} \mathrm{H}_{8} \mathrm{O}_{3}$. Loss of methyl group during formation of $\mathrm{MS}^{2}$ ion at $\mathrm{m} / z, 136$, and further elimination of $\mathrm{CO}_{2}$ during formation of a base peak ion at $m / z 92$ in $\mathrm{MS}^{3}$ spectra, suggested that peak 32 belonged to methyl 4-hydroxybenzoate.

Observed deprotonated molecular ion at $\mathrm{m} / z, 179$ and $\mathrm{MS}^{2}$ ion at $\mathrm{m} / z 135$ (loss of 44 Da) indicated that compound 21 was caffeic acid. On the basis of the obtained values of [M$\mathrm{H}]^{-}$and $[\mathrm{M}+\mathrm{H}]^{+}$, as well as $\mathrm{MS}^{2}$ and $\mathrm{MS}^{3}$ fragmentation patterns (in both negative and positive modes, respectively), compounds 14, 17 and 19 were assigned as isomers of caffeoyl quinic acid or chlorogenic acid. All these compounds showed a negative product ion at $\mathrm{m} / \mathrm{z}$ 179 due to loss of a deprotonated molecule of caffeic acid, as well as positive product ion at $\mathrm{m} / \mathrm{z}$ at 165 due to loss of quinic acid residue (Misic et al., 2015). Comparing $\mathrm{MS}^{2}$ fragmentation patterns obtained in negative ionization mode with previously published data (Misic et al., 2015; Gardana et al., 2007; Gouveia and Castilho, 2011), three isomeric dicaffeoylquinic acids (compounds 40, 41 and 44) and rosmarinic acid (compound 42) were identified in our sample. The analysis in the LTQ-Orbitrap confirmed presence of $\mathbf{4 0 , 4 1}$ and 44 with $[\mathrm{M}-\mathrm{H}]^{-}$errors of $1.315 \mathrm{ppm}, 2.034 \mathrm{ppm}$ and $2.849 \mathrm{ppm}$, respectively. The ion at $\mathrm{m} / \mathrm{z}$ 353 or [M-H-162] $]^{-}$corresponded to loss of a caffeic acid unit, while ion at $\mathrm{m} / z .191$ indicated deprotonated quinic acid. Compound 39 ( $\mathrm{t}_{r} 6.97 \mathrm{~min}$ ) gave an [M-H] ${ }^{-}$ion at $\mathrm{m} / \mathrm{z} 473$ (error $1.886 \mathrm{ppm}$ ). The $\mathrm{MS}^{2}$ fragmentation gave a fragment ion at $\mathrm{m} / z 311$ (loss of $162 \mathrm{Da}$ ) as deprotonated caftaric acid, base peak ion at $\mathrm{m} / \mathrm{z} 293$ (neutral loss of caffeic acid) and ion at $m / z$ 179, which corresponds to deprotonated caffeic acid (Figure 3 and Figure 4). Thus compound 39 was characterized as cichoric acid. 


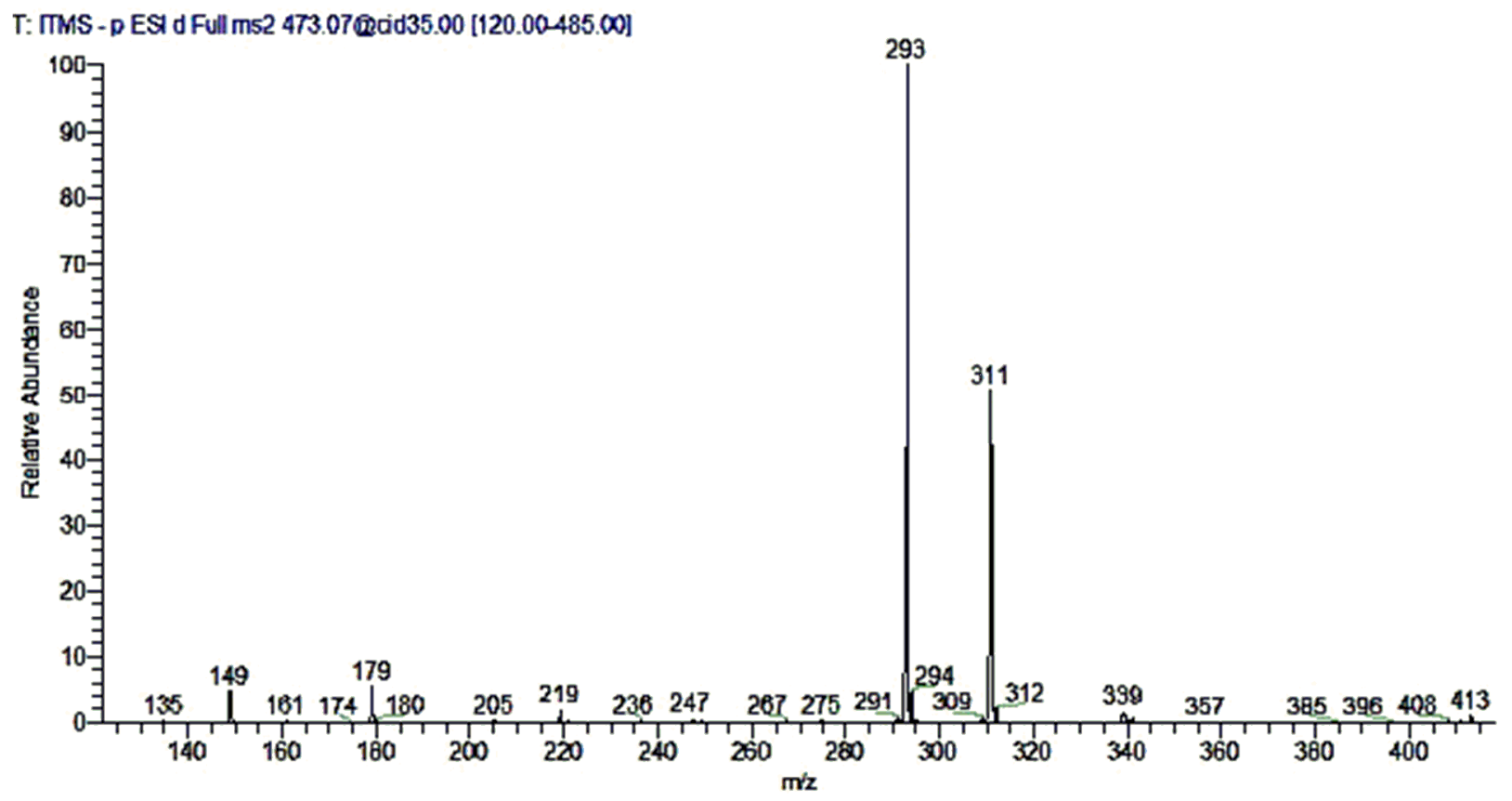

Figure 3. The $\mathrm{MS}^{2}$ spectra in negative mode of cichoric acid.
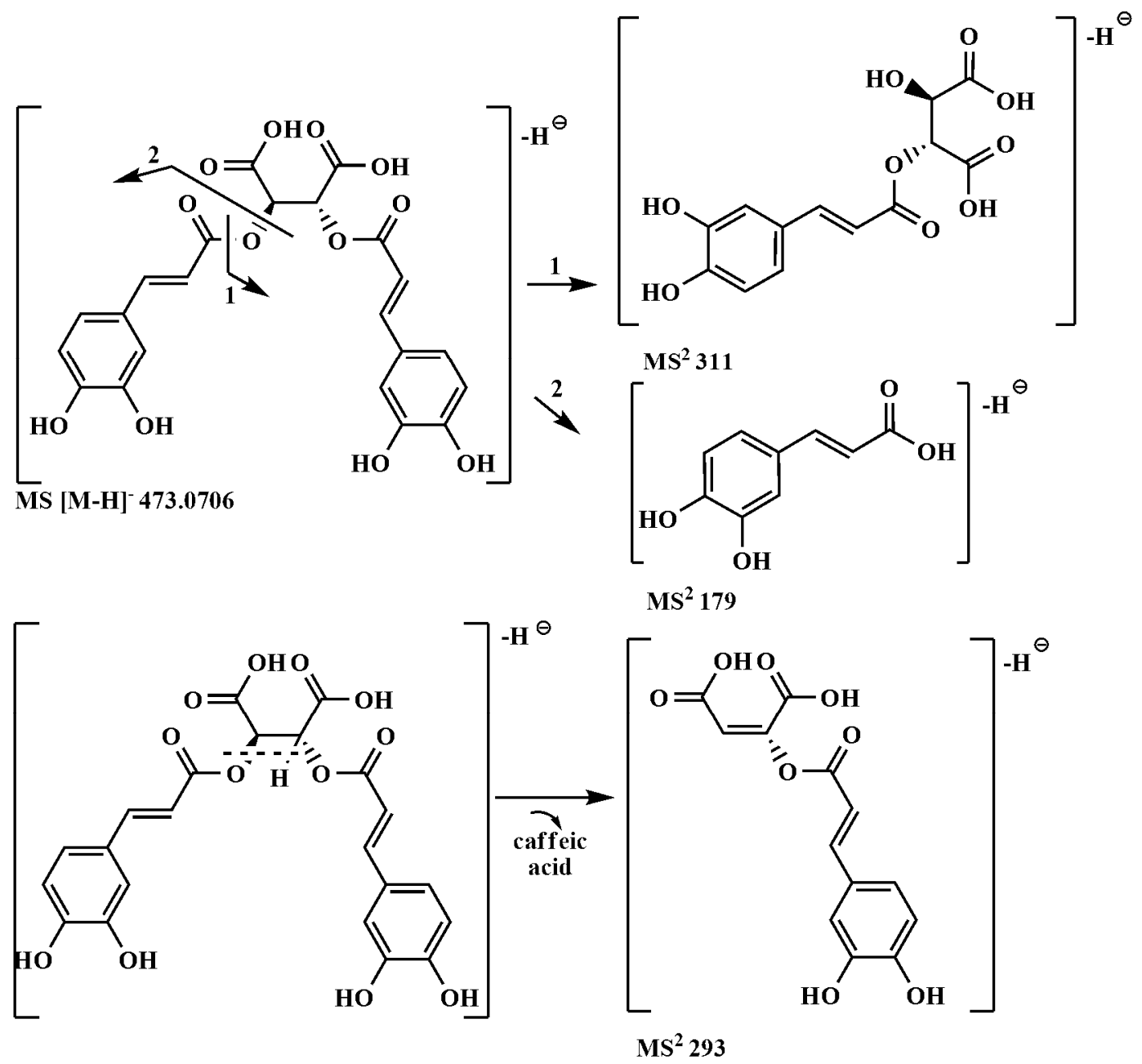

Figure 4. Possible fragmentation pathways of cichoric acid. 
Peak 28 with $[\mathrm{M}-\mathrm{H}]^{-}$at $\mathrm{m} / z, 521$ (error $-1.165 \mathrm{ppm}$ ) and $\mathrm{MS}^{2}$ ion at $m / z, 359$ as deprotonated compound 42 [M-H-glucose] was characterized as 3'-O-glucopyranosyl rosmarinic acid. By showing [M-H] $]^{-}$ion at $m / z$ 325, as well as $\mathrm{MS}^{2}$ ion at $m / z 163$ (-162 Da glucosyl residue), compound 18 was identified as 4-O-glucosyl-p-coumaric acid. Compounds 30 ( $p$-coumaric acid) and 24 (5-O-p-coumaroylquinic acid) exhibited $[\mathrm{M}-\mathrm{H}]^{-}$at $\mathrm{m} / \mathrm{z} 163$ and at $\mathrm{m} / \mathrm{z} 337$, respectively. The typical loss of $\mathrm{CO}_{2}$ was observed for compound 30 ( $\mathrm{MS}^{2}$ base peak ion at $\mathrm{m} / \mathrm{z}$ 119), while compound 24 in the $\mathrm{MS}^{2}$ spectra revealed peaks at $\mathrm{m} / \mathrm{z} 191$ and 163 corresponding to the deprotonated quinic acid [M-H-146] $]^{-}$and coumaric acid [M-H-174] respectively. Two isomeric feruoylquinic acids (compounds 25 and 26) with $[\mathrm{M}-\mathrm{H}]^{-}$at $\mathrm{m} / \mathrm{z}$ 337 were detected at $\mathrm{t}_{r} 5.76 \mathrm{~min}$ and $\mathrm{t}_{r} 5.96 \mathrm{~min}$, respectively. In both cases, $\mathrm{MS}^{2}$ of $\mathrm{m} / z .337$ showed a base ion peak at $\mathrm{m} / \mathrm{z} 191$ corresponding to the formation of deprotonated quinic acid.

\section{Flavonoid aglycones and their derivatives}

Among twenty-eight compounds, four aglycones and twenty-four flavonoid derivatives were identified in methanol extract of E. amethystinum.

Peak $51\left(\mathrm{t}_{r} 8.46 \mathrm{~min},[\mathrm{M}-\mathrm{H}]^{-}\right.$at $\mathrm{m} / \mathrm{z}$ 301, error $\left.0.756 \mathrm{ppm}\right)$ was identified as luteolin. Observed fragment ions are at $m / z, 241\left[\mathrm{M}-\mathrm{H}-\mathrm{CO}_{2}\right]^{-}, 199\left[\mathrm{M}-\mathrm{H}-\mathrm{C}_{2} \mathrm{H}_{2} \mathrm{O}-\mathrm{CO}_{2}\right]^{-}$and $175[\mathrm{M}-\mathrm{H}-$ $\left.\mathrm{C}_{3} \mathrm{O}_{2}-\mathrm{C}_{2} \mathrm{H}_{2} \mathrm{O}\right]^{-}$. Compound 52 (quercetin) yielded an $[\mathrm{M}-\mathrm{H}]^{-}$at $\mathrm{m} / \mathrm{z} 301$, while $\mathrm{MS}^{2}$ fragmentation gave fragment ions from retro-Diels-Alder (RDA) reaction at $\mathrm{m} / z, 179\left[{ }^{1,2} \mathrm{~A}\right]^{-}$, $151\left[^{1,2} \mathrm{~A}-\mathrm{CO}\right]^{-}$and $121\left[^{1,2} \mathrm{~B}\right]^{-}$(Fabre et al., 2001). Flavonol kaempferol was identified as peak 54 at $\mathrm{t}_{r} 9.50 \mathrm{~min}$, by comparing literature data with deprotonated ion at $\mathrm{m} / \mathrm{z} 285$, as well as $\mathrm{MS}^{2}$ ions at $m / z 257$ [M-H-CO $^{-}, 229$ [M-H-2CO $^{-}, 213\left[\mathrm{M}-\mathrm{H}-\mathrm{CO}_{2}-\mathrm{CO}\right]^{-}$and $\left.151{ }^{1,2} \mathrm{~A}\right]^{-}$ (Fabre et al., 2001). Presence of isorhamnetin (compound 45) was confirmed by its $\mathrm{MS}^{\mathrm{n}}$ spectra in positive ionization mode. $\mathrm{MS}^{2}$ of $[\mathrm{M}+\mathrm{H}]^{+}$at $\mathrm{m} / z 317$ (error $-1.322 \mathrm{ppm}$ ) showed peak at $\mathrm{m} / \mathrm{z} 302$, which corresponds to loss of methyl group $\left(\mathrm{CH}_{3}\right)$. Further fragmentation showed ions at $\mathrm{m} / \mathrm{z} 301$ (loss of $\mathrm{H}$ ), $274\left[\mathrm{M}+\mathrm{H}-\mathrm{CH}_{3}-\mathrm{CO}\right]^{+}, 256\left[\mathrm{M}+\mathrm{H}-\mathrm{CH}_{3}-\mathrm{CO}-\mathrm{H}_{2} \mathrm{O}\right]^{+}$and $228\left[\mathrm{M}+\mathrm{H}-\mathrm{CH}_{3}-\mathrm{C}_{2} \mathrm{O}_{2} \mathrm{H}-\mathrm{OH}\right]^{+}$, as well as two RDA fragmentation ions at $\mathrm{m} / \mathrm{z} 152\left[{ }^{1,3} \mathrm{~A}\right]^{+}$and $124\left[{ }^{1,3} \mathrm{~A}\right]^{+}$.

Three quercetin glycosides-23, 27 and 31 were found in our sample. Quercetin-3-Orutinoside (23) with an error of $1.722 \mathrm{ppm}$ yielded $[\mathrm{M}-\mathrm{H}]^{-}$ion at $\mathrm{m} / \mathrm{z} 609$. The $\mathrm{MS}^{2}$ fragmentation gave ions at $\mathrm{m} / z 463$ (loss of $164 \mathrm{Da}$, rhamnosyl residue) and aglycone fragment ion of deprotonated quercetin $\left(\mathrm{Y}_{\mathrm{O}^{-}}\right)$at $\mathrm{m} / \mathrm{z} 301$ (loss of $162 \mathrm{Da}$, glucose residue). Compound 27, quercetin-3-O-neohesperidoside, was observed both in negative and positive ionization modes, respectively. The molecular ions were at $\mathrm{m} / \mathrm{z} 609 / 611$ (error $0.310 \mathrm{ppm} /-$ $1.229 \mathrm{ppm}$ ). $\mathrm{MS}^{2}$ in negative mode showed ion at $\mathrm{m} / \mathrm{z} 301$ (loss of $308 \mathrm{Da}$, sugar residue), while $\mathrm{MS}^{3}$ fragment ions confirmed presence of quercetin moiety. Further, presence of neohesperoside residue was confirmed by $\mathrm{MS}^{2}$ fragment ions in positive mode. Observed ion at $m / z, 465$ corresponds to quercetin-3-O-glucoside (-146 Da indicated on rhamnose part), while base peak ion at $\mathrm{m} / \mathrm{z} 303$ was protonated quercetin $\left(\mathrm{Y}_{\mathrm{O}^{+}}\right)(-308 \mathrm{Da}$, neohesperidoside residue). Quercetin-3-O-glucoside (compound 31) with $[\mathrm{M}-\mathrm{H}]^{-} /[\mathrm{M}+\mathrm{H}]^{+}$at $m / z$, 463/465 (error $-2.510 \mathrm{ppm} /-1.446 \mathrm{ppm})$ gave origin to quercetin aglycone, by loss of glucose moiety $(\mathrm{m} / \mathrm{z}$ 301 in negative mode; $\mathrm{m} / \mathrm{z} 303$ in positive mode) (Aaby et al., 2012). Two quercetin derivatives (compounds $\mathbf{5 3}$ and 55) with observed $[\mathrm{M}+\mathrm{H}]^{+}$ions at $m / z 757$ (errors $-1.996 \mathrm{ppm}$ and $-1.507 \mathrm{ppm}$, respectively) and similar $\mathrm{MS}^{2}$ and $\mathrm{MS}^{3}$ spectra were identified as quercetin3-O-(di-p-coumaroyl)-glucoside isomers. The $\mathrm{MS}^{2}$ mass spectra showed base peak ion at $\mathrm{m} / \mathrm{z}$ 455 , which corresponds to loss of quercetin $[\mathrm{M}+\mathrm{H}-302]^{+}$and ion at $m / z 437[\mathrm{M}+\mathrm{H}$-quercetin- 
$\left.\mathrm{H}_{2} \mathrm{O}\right]^{+}$. The $\mathrm{MS}^{3}$ mass spectra displayed ion at $\mathrm{m} / z$ 437, indicating neutral loss of $\mathrm{H}_{2} \mathrm{O}$, ion at $\mathrm{m} / z 291$ which corresponds to coumaroyl glucoside residue (loss of $164 \mathrm{Da}$ or $p$-coumaric acid), and ion at $\mathrm{m} / \mathrm{z}, 147$ which confirmed presence of coumaroyl moiety (Figure 5).
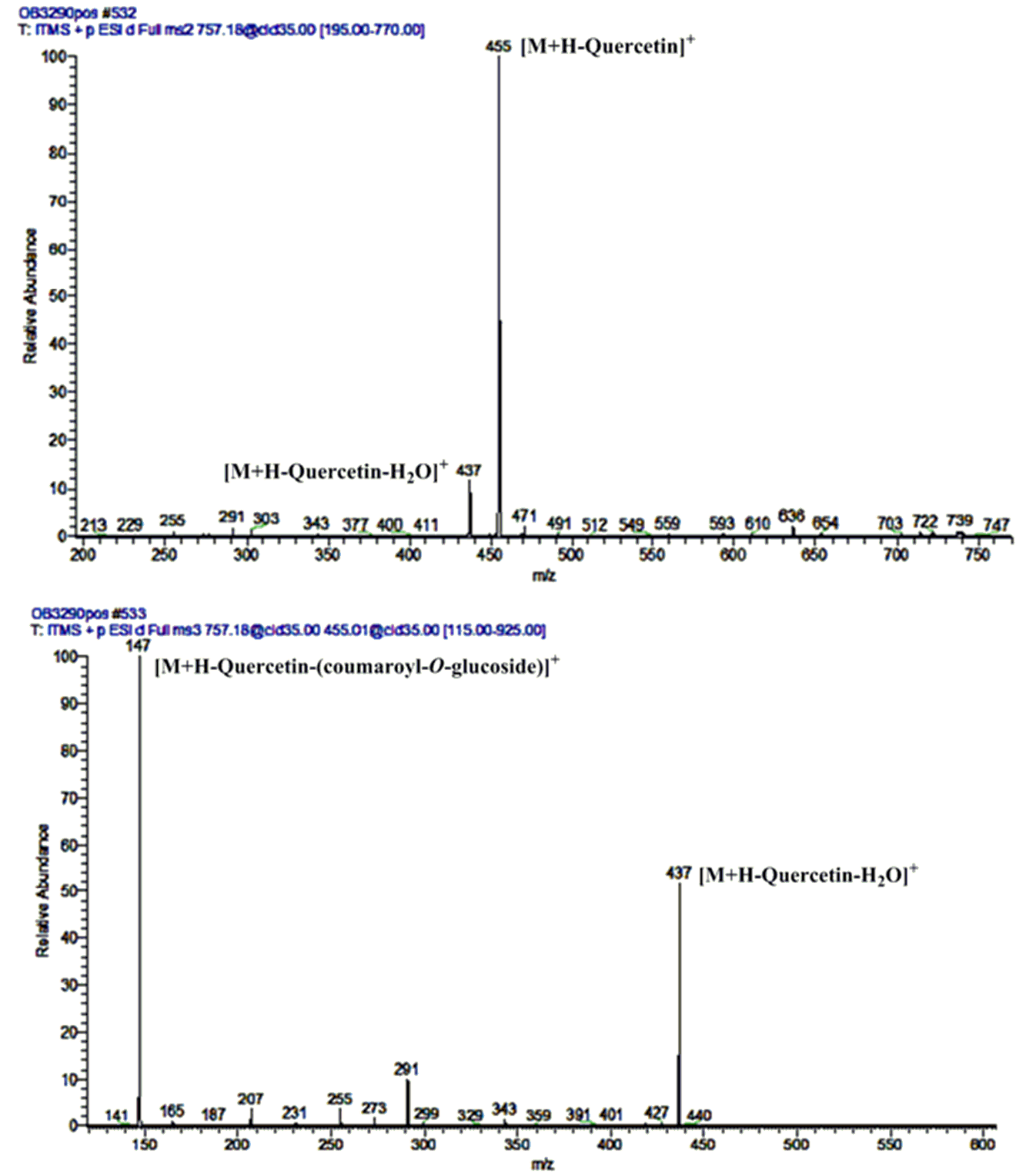

Figure 5. The $\mathrm{MS}^{2}$ and $\mathrm{MS}^{3}$ spectra in positive mode of quercetin-3-O-(di-p-coumaroyl)-glucoside.

Compound 29 exhibited an $[\mathrm{M}-\mathrm{H}]^{-} /[\mathrm{M}+\mathrm{H}]^{+}$at $\mathrm{m} / \mathrm{z}$ 593/595 (error $-0.129 \mathrm{ppm} /-1.086$ $\mathrm{ppm})$. The presence of neohesperoside residue was confirmed by $\mathrm{MS}^{2}$ fragment ions. Observed ions at $m / z$ 447/449 correspond to kaempferol-3-O-glucoside (-146 Da indicated on rhamnose part), while base peak ions at $\mathrm{m} / \mathrm{z}, 285 / 287$ correspond to kaempferol residue (loss of $308 \mathrm{Da}$, neohesperidoside residue). Compound 33 gave $[\mathrm{M}+\mathrm{H}]^{+}$ion at $\mathrm{m} / z, 565$ and in the 
$\mathrm{MS}^{2}$ fragmentation a neutral loss of $146 \mathrm{Da}$ (rhamnosyl residue) was observed to produce a fragment ion at $m / z$ 419. Similarly, loss of xylosyl residue (-132 Da) during formation of ion at $\mathrm{m} / z 433$ was found. With the facts that $\mathrm{MS}^{2}$ and $\mathrm{MS}^{3}$ spectra showed protonated ions from kaempferol, and that under used experimental condition it was not possible to determine substitution of flavonoid hydroxyl groups, compound $\mathbf{3 3}$ was tentatively identified as kaempferol- $O$-rhamnoside- $O$-xyloside. Compound 34, kaempferol-3- $O$-glycoside, displayed $[\mathrm{M}-\mathrm{H}]^{-/} /[\mathrm{M}+\mathrm{H}]^{+}$ions at $m / z 447 / 449$, as well as characteristic $\mathrm{MS}^{2}$ fragmentation ions (in negative and positive modes) at $\mathrm{m} / \mathrm{z}$ 285/287 (-162 Da, loss of glucose residue) (Ornelas-Paz et al., 2013). Another kaempferol glycoside (compound 46) was tentatively identified as kaempferol-3-O-pentoside. Its $[\mathrm{M}-\mathrm{H}]^{-/} /[\mathrm{M}+\mathrm{H}]^{+}$ions were observed at $\mathrm{m} / \mathrm{z} 417 / 419$ (error, $3.916 \mathrm{ppm} /-1.344 \mathrm{ppm}$ ), while neutral loss of $132 \mathrm{Da}$ during formation of $\mathrm{MS}^{2}$ fragment ions at $\mathrm{m} / z$ 285/287 indicated pentose residue. Compounds $47\left(\mathrm{t}_{r} 8.19 \mathrm{~min}\right)$ and $\mathbf{5 0}\left(\mathrm{t}_{r} 8.38 \mathrm{~min}\right)$ exhibited the same $[\mathrm{M}+\mathrm{H}]^{+}$at $m / z 595$ (errors, $-1.575 \mathrm{ppm}$ and $-1.474 \mathrm{ppm}$, respectively). The $\mathrm{MS}^{2}$ fragmentation lead to a base peak ion at $\mathrm{m} / \mathrm{z} 287$ due to loss of 308 Da which was characterized as a combined loss of $162 \mathrm{Da}+146 \mathrm{Da}$ proved by the $[\mathrm{M}+\mathrm{H}-146]^{+}$ion at $\mathrm{m} / \mathrm{z}$ 449. The high retention time of these compounds suggests that the neutral loss of $146 \mathrm{Da}$ is due to a coumaroyl group rather than a rhamnose unit. The ion at $m / z 287$ corresponds to the protonated aglycone ion of kaempferol, while intense fragment ion at $\mathrm{m} / \mathrm{z}, 309$ was assigned as coumaroyl-O-glucoside part (HANHINEVA et al., 2008). Compounds 48 and 49, kaempferol-3$O$-(feruloyl)-glucoside isomers, showed protonated ion of ferulic acid at $\mathrm{m} / \mathrm{z} 195$, as well as ion of kaempferol at $\mathrm{m} / \mathrm{z}, 287$. Again, $\mathrm{MS}^{2}$ fragment ion at $\mathrm{m} / \mathrm{z} 339$ belongs to feruloyl-Oglucoside. Three kaempferol-3-O-(di- $p$-coumaroyl)-glucoside isomers (compounds 56, 58 and 61) were observed at $\mathrm{t}_{r} 9.73 \mathrm{~min}, 9.92 \mathrm{~min}$ and $10.12 \mathrm{~min}$, respectively. $\mathrm{MS}^{2}$ fragment ion at $\mathrm{m} / \mathrm{z}$ at 287 indicated presence of kaempferol. The $\mathrm{MS}^{2}$ base peak ion at $m / z 455[\mathrm{M}+\mathrm{H}-$ kaempferol $]^{+}$corresponds to the di- $p$-coumaroyl- $O$-glucoside residue. The presence of one coumaroyl residue was confirmed by formation of $\mathrm{MS}^{3}$ base peak ion at $m / z 147$, while neutral loss of $308 \mathrm{Da}$ (coumaroyl-O-glucoside) $\left[\mathrm{M}+\mathrm{H}\right.$-kaempferol-308] ${ }^{+}$indicated occurrence of other coumaroyl residue. Compounds 57 and 59 gave the same $[\mathrm{M}+\mathrm{H}]^{+}$at $\mathrm{m} / \mathrm{z}$ 771 (errors, $-1.428 \mathrm{ppm}$ and $-1.830 \mathrm{ppm}$, respectively). The $\mathrm{MS}^{2}$ spectra showed base peak ion at $m / z 485[\mathrm{M}+\mathrm{H}-286]^{+}$, indicating the neutral loss of kaempferol. Further fragmentations of ion at $\mathrm{m} / z, 485$ lead to the formation of $\mathrm{MS}^{3}$ ions at $\mathrm{m} / z 177$ (-308 Da, coumaroyl-Oglucoside part) and $m / z 147$ (-338 Da, feruloyl- $O$-glucoside part), indicating occurrence of feruloyl and coumaroyl residues, respectively. Thus, those two isomers were identified as kaempferol-3-O-(p-coumaroyl)-(feruloyl)-glucosides. Compound 35 exhibited $[\mathrm{M}-\mathrm{H}]^{-}$ion at $\mathrm{m} / \mathrm{z} 623$ (error $1.154 \mathrm{ppm}$ ) showing base peak at $\mathrm{m} / z 315$ (isorhamnetin aglycone), by loss of rutinoside residue $(-308 \mathrm{Da})$. Hence, this compound was tentatively identified as isorhamnetin- $O$-rutinoside. Compounds 36 and 43 showed $[\mathrm{M}-\mathrm{H}]^{-/}[\mathrm{M}+\mathrm{H}]+$ ions at $\mathrm{m} / \mathrm{z}$ $477 / 479$ and 447/449, respectively, and losses of different sugar moieties (-162 Da, glucoside residue; -132 $\mathrm{Da}$, pentoside residue) resulting in isorhamnetin aglycone $(\mathrm{m} / \mathrm{z} 315 / 317)$ (Rivera-Pastrana, Yahia, \& Gonzalez-Aguilar, 2010). Compound 62 gave $[\mathrm{M}+\mathrm{H}]^{+}$ion at $\mathrm{m} / \mathrm{z}$ 801 (error $-2.067 \mathrm{ppm}$ ). The $\mathrm{MS}^{2}$ spectra showed base peak ion at $\mathrm{m} / \mathrm{z} 485$ (coumaroylferuloyl- $O$-glucoside), corresponding to the loss of isorhamnetin. Further fragmentations lead to the formation of ion at $m / z 467\left[\mathrm{M}+\mathrm{H} \text {-isorhamnetin- } \mathrm{H}_{2} \mathrm{O}\right]^{+} . \mathrm{MS}^{3}$ base peak ion at $m / z 177$ corresponds to the feruloyl residue, while neutral loss of $308 \mathrm{Da}$ confirmed presence of coumaroyl- $O$-glucoside part. Thus, compound 62 was identified as isorhamnetin- $O-(p$ coumaroyl)-(feruloyl)-glucoside.

Compound 37 exhibited an $[\mathrm{M}+\mathrm{H}]^{+}$ion at $m / z 509$ (error -1.566). The $\mathrm{MS}^{2}$ fragmentation revealed a neutral cleavage of $162 \mathrm{Da}$ (glucoside residue), leading to the fragment ion at $\mathrm{m} / \mathrm{z} 347$, which corresponds to the protonated molecule of syringetin. Thus, this compound was identified as syringetin- $O$-glucoside. Compound $\mathbf{3 8}$ was characterized as 
luteolin-7-O-glucoside. This compound showed an $[\mathrm{M}-\mathrm{H}]^{-}$ion at $\mathrm{m} / z$ 447 and the $\mathrm{MS}^{2}$ experiment resulted in a neutral loss of $162 \mathrm{Da}$ (ion at $\mathrm{m} / \mathrm{z} 285$ ) and typical fragment of luteolin.

\section{Coumarin derivatives}

Compound 15 (esculetin-6-O-glucoside, $\mathrm{t}_{r} 4.72 \mathrm{~min}$ ) displayed a deprotonated molecular ion at $\mathrm{m} / z, 339$ and $\mathrm{MS}^{2}$ fragmentation gave a neutral fragment of $162 \mathrm{Da}$ (glucoside residue), forming the aglycone ion $\left(\mathrm{Y}_{\mathrm{O}^{-}}\right.$) at $\mathrm{m} / \mathrm{z} 177$ (esculetin). Scopoletin-7-Oglucuronide was identified as compound 16; $[\mathrm{M}-\mathrm{H}]-$ at $\mathrm{m} / z$, 367 (error of $-0.145 \mathrm{ppm}$ ). The $\mathrm{MS}^{2}$ spectra showed deprotonated ion of scopoletin $(\mathrm{m} / \mathrm{z}, 191)$, while neutral loss of $176 \mathrm{Da}$ corresponded to the glucuronide residue. (4-Methylumbelliferone)-7-O-glucoside (compound 22) was identified in negative and positive ionization modes. Molecular ions were observed at $\mathrm{m} / \mathrm{z} 337$ (error $1.828 \mathrm{ppm}$ ) and at $\mathrm{m} / \mathrm{z} 339$ (error $-0.867 \mathrm{ppm}$ ), respectively. The $\mathrm{MS}^{2}$ spectra showed neutral loss of $162 \mathrm{Da}$, which indicated presence of glucoside residue, while ions at $\mathrm{m} / \mathrm{z}$, 175/177 confirmed presence of 4-methylumbelliferone.

\section{Other compounds}

The analysis in the LTQ-Orbitrap confirmed presence of five organic acids $(\mathbf{1}, \mathbf{2}, \mathbf{3}, \mathbf{4}$ and 6). Gluconic acid (compound 1) gave a $[\mathrm{M}-\mathrm{H}]^{-}$ion at $\mathrm{m} / \mathrm{z} 195$ (error $-1.482 \mathrm{ppm}$ ) and characteristic $\mathrm{MS}^{2}$ fragment ions at $m / z 177\left[\mathrm{M}-\mathrm{H}-\mathrm{H}_{2} \mathrm{O}\right]^{-}, \mathrm{m} / z 159\left[\mathrm{M}-\mathrm{H}-2 \mathrm{H}_{2} \mathrm{O}\right]^{-}$, and $m / z 129$ $\left[\mathrm{M}-\mathrm{H}-2 \mathrm{H}_{2} \mathrm{O}-\mathrm{CH}_{2} \mathrm{O}\right]^{-}$. Compound 2 with deprotonated molecular ion at $\mathrm{m} / z, 191$ and fragment ion at $m / z 127$ (loss of $\mathrm{CO}$ and $\mathrm{H}_{2} \mathrm{O}$ ) was identified as quinic acid. Peak $\mathbf{3}$, characterized as malic acid, showed an $[\mathrm{M}-\mathrm{H}]^{-}$at $\mathrm{m} / z 133$ (error $0.979 \mathrm{ppm}$ ), with an $\mathrm{MS}^{2}$ ion at $\mathrm{m} / z 115$ corresponding to the loss of water. Neutral loss of $\mathrm{C}_{2} \mathrm{H}_{2} \mathrm{O}_{2}\left(\mathrm{~m} / \mathrm{z}\right.$ at 59) from the $[\mathrm{M}-\mathrm{H}]^{-}$at $\mathrm{m} / \mathrm{z}$ 117 confirmed presence of succinic acid (compound 4). Peak 6, with a $[\mathrm{M}-\mathrm{H}]^{-}$at $\mathrm{m} / z 161$ (error $1.243 \mathrm{ppm}$ ), was identified as hydroxymethylglutaric acid, and its $\mathrm{MS}^{2}$ spectra showed ion at $\mathrm{m} / z, 117$ (loss of $44 \mathrm{Da}, \mathrm{CO}_{2}$ ). Compound 5 corresponded to hydroquinone- $O$-glucoside $\left([\mathrm{M}-\mathrm{H}]^{-} \text {at } m / z \text { 271) with a fragmentation pattern at } m / z, 109 \text { [M-H-glucose] }\right]^{-}$Compound 20, which showed protonated molecular ion at $\mathrm{m} / z, 469$ (error $-1.360 \mathrm{ppm}$ ), was tentatively identified as nonyl- $O$-maltoside. $\mathrm{MS}^{2}$ spectra revealed loss of $\mathrm{H}_{2} \mathrm{O}(\mathrm{m} / \mathrm{z} 451)$ and glucoside residue $(\mathrm{m} / \mathrm{z}, 307)$. On the basis of observed $[\mathrm{M}+\mathrm{H}]^{+}$ions, as well as $\mathrm{MS}^{\mathrm{n}}$ spectra, compounds 60 and 63 were characterized as dihydroxyhexadecanoic acid and hexadecanedioic acid, respectively.

\section{CONCLUSIONS}

In this study we performed for the first time UPLC-LTQ-Orbitrap-MS ${ }^{\mathrm{n}}$ non-targeted metabolic analysis of methanol extract of E. amethystinum. The approach used allowed identification of sixty-three secondary metabolites, most of them belonging to the phenolics class (simple phenolics, flavonoids and coumarins). These data suggest that this medicinal plant might be a valuable source of bioactive secondary metabolites from classes of phenolic acids, flavonoids and coumarins with beneficial proprieties, and a promising source of health products for functional food or nutraceutical industries. 


\section{Acknowledgments}

This work was financially supported by the Ministry of Education, Science and Technological Development of the Republic of Serbia (grants III41010, OI172016 and OI175039). The authors acknowledge the support of the FP7 RegPot project FCUB ERA GA No. 256716. The EC does not share responsibility for the content of the article.

\section{References:}

[1] Aaby, K., Mazur, S., Nes, A., Skrede, G. (2012): Phenolic compounds in strawberry (Fragaria $\mathrm{x}$ ananassa Duch.) fruits: Composition in 27 cultivars and changes during ripening. Food Chemistry 132 (1): 86-97. doi: 10.1016/j.foodchem.2011.10.037

[2] Calvino, C.I., Martínez, S.G., Downie, S.R. (2007): The evolutionary history of Eryngium (Apiaceae, Saniculoideae): rapid radiations, long distance dispersals, and hybridizations. Molecular Phylogenetics and Evolution 46 (3): 1129-1150. doi: 10.1016/j.ympev.2007.10.021

[3] Crowden, R.K., Harborne, J.B., Heywood, V.H. (1969): Chemosystematics of the Umbelliferae-a general survey. Phytochemistry 8 (10): 1963-1984. doi: 10.1016/S00319422(00)88084-X

[4] DraKe, D., LAM, J. (1972): Seseli acetylene from Eryngium bourgatti. Phytochemistry 11 (8): 2651-2652. doi: 10.1016/S0031-9422(00)88584-2

[5] Fabre, N., Rustan, I., DE Hoffmann, E., Quetin-LeclercQ, J. (2001): Determination of flavone, flavonol, and flavanone aglycones by negative ion liquid chromatography electrospray ion trap mass spectrometry. Journal of the American Society for Mass Spectrometry 12 (6): 707-715. doi: 10.1016/S1044-0305(01)00226-4

[6] Facciola, S. (1990): Cornucopia: A Source Book of Edible Plants. Vista, California: Kampong Publications.

[7] Flamini, G., Tebano, M., Cioni, P.L. (2008): Composition of the essential oils from leafy parts of the shoots, flowers and fruits of Eryngium amethystinum from Amiata Mount (Tuscany, Italy). Food Chemistry 107 (2): 671-674.

doi: 10.1016/j.foodchem.2007.08.064

[8] Gardana, C., Scaglianti, M., Pietta, P., Simonetti, P. (2007): Analysis of the polyphenolic fraction of propolis from different sources by liquid chromatographytandem mass spectrometry. Journal of Pharmaceutical and Biomedical Analysis 45 (3): 390-399. doi: 10.1016/j.jpba.2007.06.022

[9] Gouveia, S., CAstilho, P.C. (2011): Antioxidant potential of Artemisia argentea L`Her alcoholic extract and its relation with the phenolic composition. Food Research International 44 (6): 1620-1631. doi: 10.1016/j.foodres.2011.04.040

[10] Hanhineva, K., RogacheV, I., Kokko, H., Mintz-Oron, S., Venger, I., Karenlampi, S., Aharoni A. (2008): Non-targeted analysis of spatial metabolite composition in strawberry (Fragaria x ananassa) flowers. Phytochemistry 69 (13): 2463-2481. doi: 10.1016/j.phytochem.2008.07.009 
[11] Kartal, M., Mitaine-Offer, A.C., Paululat, T., Abu-Asaker, M., Wagner, H., MiRjolet, J.F., Guilbaud, N., LACAIlle-Dubois M.A. (2006): Triterpene saponins from Eryngium campestre. Journal of Natural Product 69 (7): 1105-1108. doi: $10.1021 / \mathrm{np} 060101 \mathrm{w}$

[12] MišIĆ, D., ŠIler, B., GAŠić, U., Avramov, S., Živković, S., NeSTOROvić ŽIvković, J., MiLUTINOvić, M., TEŠIĆ Z. (2015): Simultaneous UHPLC/DAD/(+/-)HESI-MS/MS Analysis of phenolic acids and nepetalactones in methanol extracts of Nepeta species: A possible application in chemotaxonomic studies. Phytochemical Analysis 26 (1): 72-85. doi: $10.1002 /$ pca.2538

[13] Ornelas-Paz, J.D., Yahia, E.M., Ramirez-Bustamante, N., Perez-Martinez, J.D., Escalante-Minakata, M.D., Ibarra-Junquera, V., Acosta-MuÑIZ, C., GuerreroPRIETO, V., OCHOA-REYES, E. (2013): Physical attributes and chemical composition of organic strawberry fruit (Fragaria x ananassa Duch, Cv. Albion) at six stages of ripening. Food Chemistry 138 (1): 372-381. doi: 10.1016/j.foodchem.2012.11.006

[14] Rivera-Pastrana, D.M., Yahia, E.M., GonZalez-Aguilar, G.A. (2010): Phenolic and carotenoid profiles of papaya fruit (Carica papaya L.) and their contents under low temperature storage. Journal of the Science of Food and Agriculture 90 (14): 23582365. doi: $10.1002 /$ jsfa.4092

[15] Tchoumtchoua, J., Nuamen, D., Mbanya, J.C., Skaltsounisa, A.L., Halabalaki, M. (2013): Structure-oriented UHPLC-LTQ Orbitrap-based approach as a dereplication strategy for the identification of isoflavonoids from Amphimas pterocarpoides crude extract. Journal of Mass Spectrometry 48 (5): 561-575. doi: 10.1002/jms.3167

[16] Worz, A. (2004): On the Distribution and Relationships of the South-West Asian Species of Eryngium L. (Apiaceae-Saniculoideae). Turkish Journal of Botany 28 (1-2): 85-92.

[17] Zhang, Z.Z, Li, S.Y., Ownby, S., Wang, P., Yuan, W., Zhang, W., Scott Beasley, R. (2008): Phenolic compounds and rare polyhydroxylated triterpenoid saponins from Eryngium yuccifolium. Phytochemistry 69: 2070-2080.

doi: 10.1016/j.phytochem.2008.03.020 